\title{
Transient hypertension as a complication of circular external fixator- Ilizarov frame
}

\author{
*Mihira Manamperi ${ }^{1}$, Dimuthu Tennakoon ${ }^{1}$, Tharindi Sooriyapperuma ${ }^{1}$, Thilini Somaratne ${ }^{2}$, Chathurika \\ Abeysinghe ${ }^{1}$
}

Sri Lanka Journal of Child Health, 2020; 49(3): 295-296

http://dx.doi.org/10.4038/sljch.v49i3.9153

(Key words: Hypertension, external fixator, Ilizarov frame, hemimelia),

\section{Introduction}

In children, early diagnosis of hypertension and its aetiology is essential to minimize long-term complications. In some instances, we have to look beyond the known causes of secondary hypertension to make the correct diagnosis. We describe the case of a patient where the cause of secondary hypertension was plausibly an external fixator.

\section{Case history}

A 7 year old Sri Lankan boy who was having right fibular hemimelia as a congenital malformation, underwent insertion of right sided Ilizarov frame (Figure 1).

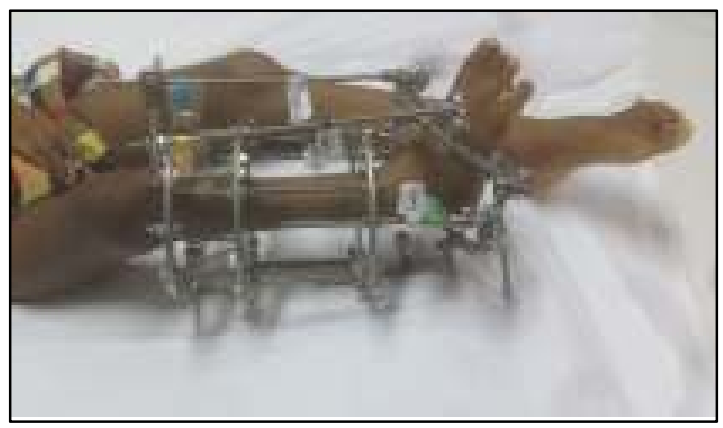

Figure 1: Patient after insertion of Ilizarov frame

He was found to have significant hypertension postoperatively despite being normotensive before and during the surgery. The systolic and diastolic blood pressures ranged between $136 \mathrm{mmHg} / 160 \mathrm{mmHg}$ and $90 \mathrm{mmHg} / 120 \mathrm{mmHg}$ respectively post operatively. His height compatible $95^{\text {th }}$ centile blood pressure values were $117 / 80 \mathrm{mmHg}$. He did not have any symptoms attributable to hypertension.

${ }^{1}$ District General Hospital Gampaha, ${ }^{2}$ Medical Research Institute, Colombo,

*Correspondence: mihira123@GMAIL.COM

orcid.org/ 0000-0002-3394-1937

(Received on 18 January 2019: Accepted after revision on 22 March 2019)

The authors declare that there are no conflicts of interest

Personal funding was used for the project.

Open Access Article published under the Creative

Commons Attribution CC-BY (CC) (P) License
Apart from the limb abnormality, past medical history was uneventful. He was developmentally normal and there was no family history of premature hypertension. His physical examination was normal apart from the repeatedly high blood pressure readings while receiving adequate pain relief. He was averagely built with no external evidence of syndromic illnesses known to be associated with secondary hypertension (e.g. Cushing's syndrome).

He was extensively investigated for secondary hypertension. The full blood count, serum electrolytes, renal function tests, urinalysis, erythrocyte sedimentation rate, thyroid function tests and serum ionized calcium levels were within their respective reference ranges. The ultrasound scan of the abdomen with Doppler studies ruled out suprarenal masses or renal artery stenosis. A 24 hour urinary vanillyl mandelic acid (VMA) and metanephrine levels and serum cortisol were also within normal limits. Echocardiography ruled out a coarctation of the aorta as well as left ventricular hypertrophy. The latter feature along with the absence of any retinal changes ruled out long standing hypertension.

Since the blood pressure readings were well above the $95^{\text {th }}$ centile, the patient was started on low-dose of nifedipine three times a day with superadded prazosin. These medications helped to bring down the blood pressure values towards the $95^{\text {th }}$ centile for his height. Since the initial work up did not help to arrive at a diagnosis evaluation of renin and aldosterone levels were on the cards despite his normal serum electrolytes values and the lack of metabolic alkalosis.

At this point the medical team decided to look for rare causes of hypertension, particularly those related to the recent surgery. There was a single case report of a child who was found to have high blood pressure after the application of a Taylor spatial frame (another form of circular external fixator for mid-shaft of tibia), after a road traffic accident. Several months later once the circular external fixator was removed, blood pressure reverted to the normal level ${ }^{1}$. However, In our patient, we managed to tail off nifedipine and then prazosin while having 
the external fixator in situ. He reached normal blood pressure reading 4 weeks after surgery.

\section{Discussion}

The association of hypertension with limb lengthening surgeries and application of an external fixator has been reported infrequently in the past. The pathophysiologic basis of hypertension in relation to the circular external fixators is not fully understood. It has been hypothesized that during the limb lengthening surgeries this phenomenon occurs secondary to the stretching of the sciatic nerve which could give rise to reflex vasoconstriction. When it comes to circular external fixators, hypertension may occur from the irritation of the nerve due to the changing the microenvironment of the limb leading to reflex vasoconstriction and subsequently hypertension ${ }^{1}$. An extensive retrospective study done in United Kingdom to evaluate the association between hypertension versus external fixators concluded that $12.2 \%(5 / 41)$ of cases had stage 2 hypertension while on an external fixator (stage two hypertension defined as systolic or diastolic BP $\geq$ $99^{\text {th }}$ centile plus $\left.5 \mathrm{mmHg}\right)^{2}$.

This case report highlights the importance of keeping this complication in mind when evaluating children for hypertension after an orthopaedic procedure such as application of circular external fixators. Routine blood pressure monitoring during the preoperative period helps to establish a baseline blood pressure for the child. Awareness of the association of hypertension following insertion of a circular external fixator, will reduce the need for extensive investigations for causes of secondary hypertension in children. Anticipation of this complication will enable timely commencement of antihypertensive medications in instances where the fixator cannot be removed and this will avoid the risk of complications of paediatric hypertension which otherwise has been reported previously ${ }^{2}$.

\section{References}

1. Changulani M, Bradbury M, Zenios M. Hypertension as a complication of Taylor spatial frame. Journal of Pediatric Orthopedics B 2009; 18(6):392-3.

https://doi.org/10.1097/BPB.0b013e32832

dbecf

PMid: 19623085

2. Oroko MD, Wadia FD, Farrell R, Bradbury $\mathrm{M}$, Zenios M. Hypertension as a complication of circular external fixators, Journal of Pediatric Orthopaedics B 2013, 22(3):270-4.

https://doi.org/10.1097/BPB.0b013e32835

ec5a6

PMid: 23443142 